\title{
Review Article \\ Physiological Roles of Class I HDAC Complex and Histone Demethylase
}

\author{
Tomohiro Hayakawa and Jun-ichi Nakayama \\ Laboratory for Chromatin Dynamics, RIKEN Center for Developmental Biology, 2-2-3 Minatojima-minamimachi, \\ Chuo-ku, Kobe 650-0047, Japan
}

Correspondence should be addressed to Jun-ichi Nakayama, jnakayam@cdb.riken.jp

Received 15 July 2010; Accepted 7 September 2010

Academic Editor: Minoru Yoshida

Copyright ( $(2011$ T. Hayakawa and J.-i. Nakayama. This is an open access article distributed under the Creative Commons Attribution License, which permits unrestricted use, distribution, and reproduction in any medium, provided the original work is properly cited.

\begin{abstract}
Epigenetic gene silencing is one of the fundamental mechanisms for ensuring proper gene expression patterns during cellular differentiation and development. Histone deacetylases (HDACs) are evolutionally conserved enzymes that remove acetyl modifications from histones and play a central role in epigenetic gene silencing. In cells, HDAC forms a multiprotein complex (HDAC complex) in which the associated proteins are believed to help HDAC carry out its cellular functions. Though each HDAC complex contains distinct components, the presence of isoforms for some of the components expands the variety of complexes and the diversity of their cellular roles. Recent studies have also revealed a functional link between HDAC complexes and specific histone demethylases. In this paper, we summarize the distinct and cooperative roles of four class I HDAC complexes, Sin3, NuRD, CoREST, and NCoR/SMRT, with respect to their component diversity and their relationship with specific histone demethylases.
\end{abstract}

\section{Introduction}

Eukaryotic chromosomes consist of two cytologically defined structures, euchromatin, and heterochromatin. Euchromatin contains transcribable genes, which are subject to either activation or inactivation depending on the cellular situation, whereas heterochromatin contains transcriptionally inert genes. Structural changes in chromatin are tightly linked with posttranscriptional modifications of the histone tails. The combinations of histone modifications establish local and global patterns of chromatin structure and define specific downstream events [1]. These patterns are highly dynamic and can be altered by multiple extracellular and intracellular stimuli. Thus, chromatin has been proposed to serve as a signaling platform by which various signaling pathways are integrated.

Among the numerous covalent modifications identified so far, acetylation and methylation play central roles in chromatin dynamics [2]. Histone acetylation on lysine residues is primarily associated with gene activation, and its levels are controlled by two counteracting enzymes: histone acetyltransferases (HATs) and histone deacetylases
(HDACs). Many lysine and arginine residues on histones are target sites of methylation. In contrast to acetylation, histone methylation is linked with both gene activation and inactivation and is regulated by histone methyltransferases (HMTs) and histone demethylases (HDMs). In most cases, these histone-modifying enzymes are present in multisubunit protein complexes, in which the other components are thought to regulate enzyme activities, modulate substrate recognition, recruit other cofactors, or carry out other undefined functions.

In metazoans, HDACs are divided into three classes, Class I to Class III, based on their sequence similarity. Class I HDACs, which include HDAC1, HDAC2, and HDAC3, are found in four distinct multiprotein complexes, the Sin3, NuRD, CoREST, and NCoR/SMRT complexes. These complexes are highly conserved and function in distinct cellular processes including cell-cycle regulation $[3,4]$, maintenance of stem cell pluripotency [5], self-renewal, and cellular differentiation. Recent studies have revealed that all of these complexes are associated with specific HDMs. The HDMs are classified into two distinct enzyme families: the nuclear amine oxidase homologs and the JmjC-domain 


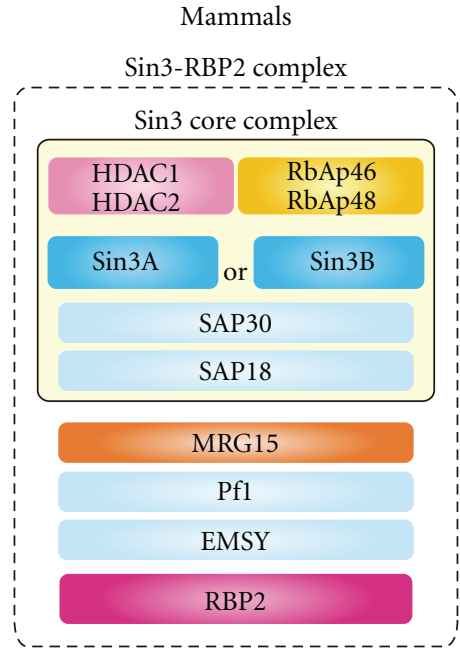

(a)

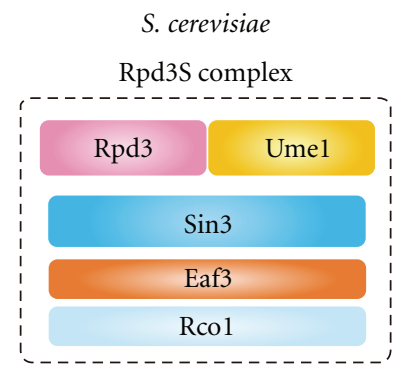

(b)

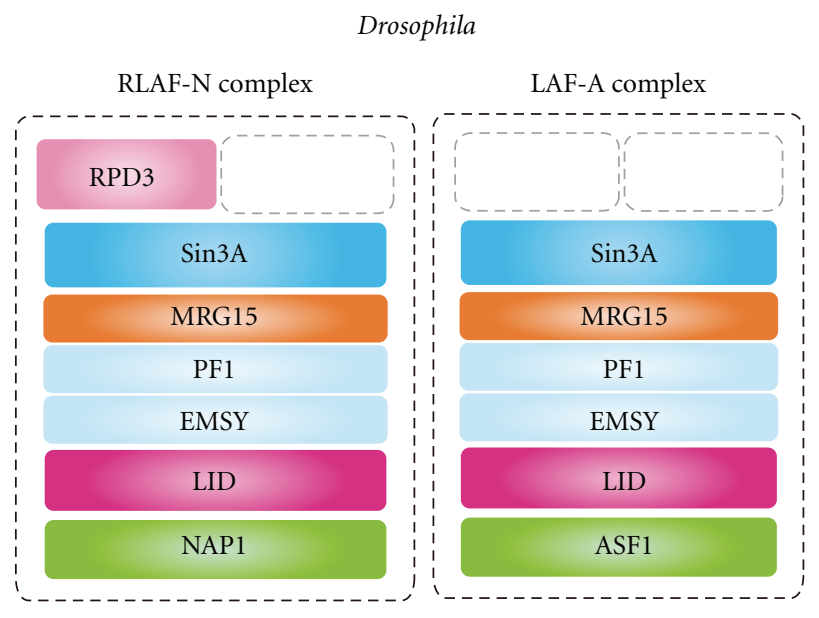

(c)

FIgURE 1: The Sin3 complexes. Schematic representation of the Sin3-containing HDAC complexes: (a) the Sin3-core and Sin3-RBP2 complexes in mammalian cells; (b) the Rpd3S complex in S. cerevisiae; (c) RLAF-N and LAF-A complexes in Drosophila. The empty dotted boxes indicate missing subunits compared with the Sin3-core complex.

proteins. LSD1 (KDM1A, also known as AOF2/BHC110) is a member of the former family and was the first histone lysine demethylase identified; it preferentially demethylates methylated histone $\mathrm{H} 3$ lysine 4 (H3K4me) [6]. The latter enzyme family, the JmjC-domain proteins, plays important roles in development and cellular differentiation [7-10]. This family has 27 members in mammals, and these members are divided into 11 subgroups based on similarity within the JmjC domain [11]. So far, seven of these subgroups have been shown to have demethylase activity for specific residues on histone $\mathrm{H} 3$ or $\mathrm{H} 4$.

To achieve strict transcriptional regulation in the complicated chromosomal architecture of the nucleus, it makes sense that eukaryotic organisms developed a system involving combinatorial histone modifications and protein complexes that contain multiple enzymatic activities, such as HDAC and HDM. It is noteworthy that cells produce a variety of $\mathrm{HDAC}$-containing complexes by replacing an integral component with one of its subtypes. These subtype-specific HDAC complexes appear to play distinct roles in particular cellular contexts. In addition, incorporating sequence-specific DNA-binding proteins, such as zinc-finger proteins, can also modulate the function of the HDAC complex. In this paper, we focus on how HDAC-HDM interactions and subtype-specificity regulate functions of the Class I HDAC-containing complexes, in transcriptional regulation and other cellular processes.

\section{The Sin3 Complex}

The HDAC-containing multiprotein complex, Sin3 complex, is highly conserved from yeast to humans. In mammalian cells, the Sin3 complex consists of six core subunits, HDAC1/2, RbAp46, RbAp48, Sin3A/Sin3B, SAP18, and
SAP30 (Figure 1(a)) [12-14]. Mammalian Sin3A and Sin3B $(\mathrm{mSin} 3 \mathrm{~A}$ and $\mathrm{mSin} 3 \mathrm{~B})$ show high sequence similarity and an overlapping expression pattern, and both are essential in mouse development. However, these subtypes play distinct and nonoverlapping roles. $\mathrm{mSin} 3 \mathrm{~A}$ has a critical role in the early developmental preimplantation stage, since no mSin3A-null zygotes are found at E6.5 [15]. mSin3Adepleted mouse embryonic fibroblasts (MEFs) exhibit the deregulation of genes involved in cell-cycle control, DNA replication, DNA repair, apoptosis, chromatin modification, and mitochondrial metabolism. Transcriptome analysis has revealed that $\mathrm{mSin} 3 \mathrm{~A}$ modulates the transcriptional network controlled by Myc-Mad, E2F, or p53. On the other hand, $\mathrm{mSin} 3 \mathrm{~B}$ has an essential function at the late-gestation stage, and its mutant is different from the mSin3A-/- embryo [16]. In particular, erythrocyte and granulocyte differentiation and G0/G1 cell-cycle control are impaired in the mSin3B-/embryo, and these phenotypes are due to the derepression of E2F target genes.

Transcriptional regulation is tightly coupled with dynamic change of histone acetylation in the promoter regions. However, the Sin3 complex functions not only at promoter regions but also at transcribed regions. In Saccharomyces cerevisiae, $\operatorname{Sin} 3$ and a Class I HDAC, Rpd3, are involved with two functionally distinct complexes, Rpd3L and Rpd3S [17]. Both complexes contain Sin3, Rpd3, and Ume1. In addition to these core subunits, Rpd3L contains at least six unique components: Rxt1, Rxt2, Dep1, Sds3, Pho23, and Sap30, whereas Rpd3S contains Rco1 and Eaf3 as unique components (Figure 1(b)) [18]. Rpd3L is localized primarily to promoter regions. In contrast, $\mathrm{Rpd} 3 \mathrm{~S}$ is localized to transcribed regions that are enriched in methylated $\mathrm{H} 3 \mathrm{~K} 36$ (H3K36me). H3K36me is a mark catalyzed by Set2 (KMT3) and is tightly coupled with the transcriptional elongation processes [19]. Rpd3S recognizes this H3K36me mark via 
a combined action of chromodomain of Eaf3 and PHD (plant homeobox domain) of Rco1 [20-22]. Deletion of Set2 or one of the Rpd3S-specific components results in spurious transcripts that emerge from incorrect transcription start sites in some transcriptionally active genes [17]. Since the $\mathrm{H} 4$ acetylation levels within transcribed regions are increased in these mutant cells, Rpd3S is thought to repress unfavorable transcription by maintaining transcribed regions in a hypoacetylated state. In metazoans, however, the relationship between $\mathrm{mSin} 3$ complexes and the emergence of aberrant transcripts remains unclear.

One of the Rpd3S-specific components, Eaf3, is highly conserved from yeast to humans. The human Eaf3 homologue MRG15 was initially identified as a factor closely related to MORF4 (mortality factor on human chromosome 4 ), whose transient expression induces senescence in a subset of human tumor cell lines [23, 24]. MRG15 is a stable component of mSin3-HDAC complexes, and the MRG15-associated mSin3-HDAC complex also contains Pf1, a component that shares similarity with $S$. cerevisiae Rcol [25], and a histone H3K4-specific demethylase, RBP2 (KDM5A) [26] (Figure 1(a)). Of note, MRG15 is shared with at least two other complexes: the Tip60 (NuA4)-HAT complex [26-28] and the BRCA2-containing DNA-damageresponsive complexes $[29,30]$. Although we will not discuss these complexes further in this paper, the MRG15-associated complexes are implicated in DNA-damage responses [29, 31-33], in addition to transcriptional regulation, suggesting that histone acetylation dynamics is tightly coupled with histone eviction/deposition during DNA-repair processes.

We previously demonstrated that MRG15 recruits RBP2 and controls the H3K4me levels on transcribed regions via the RBP2 activity [26]. In addition, van Oevelen et al. reported that the majority of Sin 3 target genes (58\%) are bound by RBP2, which spreads over the region immediately downstream of the transcription start site on a subset of E2F target genes during differentiation [34]. These results suggest that the Sin3-HDAC complex and RBP2 play a cooperative role in repressing target genes through deacetylation, demethylation, and, probably, the repositioning of nucleosomes. In S. cerevisiae, no evidence has been reported for a physical interaction or functional link between the Sin3 complex and the KDM5 homologue Jhd2. In Drosophila, RPD3, MRG15, and PF1, but not SIN3, were identified in an LID-(a KDM5 homologue) containing complex [35]. Intriguingly, the deacetylation activity of RPD3 is inhibited by its interaction with any of these associated proteins, including LID, implying that the relationship between the HDAC and HDM activities is counteractive.

Recent reports have shown that LID associates with SIN3 and functions in Notch silencing [36]. The Notch signaling pathway plays pleiotropic roles during embryonic development and is important for the regulation of cellular selfrenewal [37, 38]. Moshkin et al., purified factors associated with histone chaperones (ASF1 and NAP1) from Drosophila S2 cells or embryonic nuclear extract, and identified several peptides, including LID, SIN3A, PF1, RPD3, MRG15, and a BRCA2-binding protein, EMSY. Using a reciprocal purification approach, they further showed that NAP1 binds the RPD3 and LID-Associated Factors (RLAFs) consisting of RPD3, LID, SIN3A, PF1, EMSY, and MRG15, whereas ASF1 binds LAF, a similar complex lacking RPD3 (Figure 1(c)). Both of these complexes, RLAF-NAP1 (RLAF-N) and LAFASF1 (LAF-A), are required for the transcriptional repression of Notch-regulated genes. In agreement with the enzymatic activity of LID, the knockdown of ASF1, NAP1, or any of the LAF components results in an accumulation of $\mathrm{H} 3 \mathrm{~K} 4 \mathrm{me} 3$ at the promoter and enhancer regions. Interestingly, H3K4me3 accumulation is not observed in RPD3 knockdown cells [36]. This implies that LID recruitment to the promoter/enhancer regions of Notch target genes may not depend on the entire RPD3-containing RLAF complex. Considering that the RPD3-containing RLAF complex resembles Rpd3S in $S$. cerevisiae in its subunit composition (Figures 1(b) and 1(c)), it is possible that RLAF acts at the rest of transcribed regions, with or without the association with NAP1. This possibility will be tested by future studies.

RBP2/KDM5A was also found to be involved in the Notch pathway in a study using mammalian cells [39]. RBP-J is a nuclear effector of Notch signaling. Upon ligand binding, RBP-J activates the expression of Notch target genes, and, in the absence of Notch signal, RBP-J switches off the target gene expression. Liefke et al. showed that RBP2/KDM5A associates with RBP-J in vivo. RBP2/KDM5A is colocalized with RBP-J at the promoter region of Notch target genes and regulates their expression. Although the involvement of HDAC at the target promoter region was not described in this study, it is most likely that RBP2 works together with an HDAC complex, as in the case of the LAF-A complex in Drosophila.

Another function of the Sin 3 complex was revealed by a genome-wide study. The gene-expression profiles of Sin3-depleted Drosophila cells were analyzed by high-density oligonucleotide array [40]. This analysis revealed that the expression of $\sim 3 \%$ of the Drosophila genes is altered in Sin3-depleted cells. The affected genes are associated with diverse biological processes, including signal transduction, transcriptional regulation, and cell-cycle control. Interestingly, the list of affected genes also includes a substantial fraction of genes involved in cytosolic and mitochondrial energy-generating pathways. Furthermore, Sin3-deficient Drosophila cells exhibit an increase in mitochondrial mass. In accordance with this observation, the genome-wide analysis of RBP2-binding sites using human breast cancer cell lines revealed that RBP2 is enriched at genes encoding proteins that localize to the mitochondrion, including mitochondrial ribosomal proteins [41]. Moreover, the alteration of RBP2 leads to mitochondrial defects in human cells, as in Sin3depleted Drosophila cells.

Overall, the Sin3-HDAC complexes associated with KDM5 regulate the transcription of many genes, in processes such as Notch signaling and mitochondrial functions. Notably, the transcriptional regulation is achieved by dynamic changes in histone modifications not only at promoter regions, but also at transcribed regions. The Sin3KDM5 complex appears to play an important role at both regions by exchanging its associated cofactors. 


\section{The Mi-2/NuRD Complex}

The NuRD complex was first purified based on its histone deacetylase and nucleosome-remodeling activities $[14,42$, 43]. Although the NuRD complex has been identified in mammalian and Xenopus cells, its major components have also been found in Drosophila, C. elegans, and Arabidopsis, suggesting that the complex exists widely in animal and plant species. The biochemical association of the histone deacetylase and nucleosome-remodeling activities suggests that these two activities are functionally coupled in this complex action.

The NuRD core complex consists of HDAC1/2, RbAp46, RbAp48, CHD3/CHD4 (Mi-2), MBD2/MBD3, MTA1/ MTA2/MTA3, and p66 $\alpha / \mathrm{p} 66 \beta$ (Figure 2(a)). HDAC1/2 and RbAp46/48 are shared with the Sin3 complex. Mi-2 was first identified as an autoantigen in the human connective tissue disease dermatomyositis [44], and is a member of the SWI2/SNF2-related chromatin-remodeling ATPases, which target the chromatin region and unwind the nucleosome structure. MBD2 and MBD3 belong to the MBD (methylCpG binding) domain family although MBD3 is unable to bind methyl-CpG $[45,46]$. Since the NuRD complex can interact with both MBD2 and MBD3, it was first hypothesized to target methylated DNA through its interaction with MBD2 [47]. However, recent reports have shown that MBD3 and MBD2 are exclusively associated with the NuRD complex and form distinct complexes, MBD3/NuRD and $\mathrm{MBD} 2 / \mathrm{NuRD}$ [48]. MBD3 and MBD2 are very similar (70\% identical), but only MBD3 is essential for mouse development [49].

The MTA protein family has three members, MTA1, MTA2, and MTA3, and vertebrates have two additional splicing variants, small form of MTA1 (MTA1s) and MTA3L $[50,51]$. MTA1s, however, lacks a nuclear localization signal and is found in the cytoplasm [50]; it is therefore unlikely to be a component of nuclear Mi-2/NuRD. Among these MTAs, MTA1 and MTA3 have been clearly shown to be involved in tumor progression. Their expression is upregulated in several types of tumors, and their expression states are closely correlated with the invasive growth properties of tumors [51-53]. MTA1 is induced by the growth factor heregulin and is a potent corepressor of estrogen-receptor element-(ERE-)driven transcription [54]. Similarly, in breast epithelial cells, MTA3 is induced by estrogen, constitutes an estrogen-dependent component of the Mi-2/NuRD complex, and plays a critical role in repressing the expression of Snail, a master regulator of the epithelial-to-mesenchymal transition [51]. These data suggest that MTA proteins are mutually exclusive in the NuRD complex (Figure 2(a)) and that distinct $\mathrm{NuRD}$ complexes function as transcriptional repressors in different signaling pathways.

The NuRD complex plays important roles in development. Mice lacking a functional $M b d 3$ gene die prior to midgestation [49]. Embryonic stem (ES) cells lacking Mbd3 are viable, but they fail to completely silence genes that are expressed before implantation of the embryo [55] and are unable to commit to developmental lineages [56]. Another NuRD component, p66, was first cloned in Drosophila as a genetic modifier of the Wingless signaling pathway. The human paralogs p66 $\alpha$ (GATAD2A) and p66 $\beta$ (GATAD2B) function synergistically in transcriptional repression, and both bind to MBD2 [57]. Although p66 $\alpha$ is not required for normal blastocyst development or implantation, loss-offunction mutant embryos of p66 die at around day 10 of embryogenesis [58].

ES cells require several key molecules, such as Nanog and Oct4, for their self-renewal and pluripotency [59]. Nanogcontaining complexes were recently purified from mouse ES cells, and several NuRD-related components were identified as Nanog-interacting proteins [5]. Interestingly, the Nanoginteracting NuRD-like complex contains most of the NuRD components (Mta1, Mta2, Hdac1/2, p66 $\alpha$, and p66 $\beta$ ) but lacks Chd3/4, Mbd3, and RbAp46/Rbbp7 (Figure 2(b)). This NuRD-like unique complex was thus named NODE (for Nanog- and Oct4-associated deacetylase). Without Mbd3, deacetylase activity in the canonical NuRD complex is greatly reduced [47], but the NODE complex contains HDAC activity comparable to that of NuRD complexes. Importantly, Mbd3-knockdown ES cells show different geneexpression profiles than Mta1- or Mta2-knockdown ES cells. Moreover, while Mbd3 loss-of-function mutant ES cells can self-renew, the knockdown of NODE subunits leads to the increased expression of developmentally regulated genes and ES-cell differentiation. These observations support the idea that NODE is functionally distinct from canonical NuRD.

Although the functional interaction between NuRD/ NODE and histone demethylases in ES cells is still unknown, cooperative roles of NuRD and LSD1 have been described (Figure 2(a)) [60]. Using HeLa cells, Wang et al. purified the MTA2-containing protein complexes and identified LSD1 in addition to other NuRD components [60]. LSD1 interacts directly with all three MTA proteins and appears to form distinct complexes. The NuRD-LSD1 complexes target distinct, yet overlapping, sets of genes. Transcriptional analyses have revealed that these complexes regulate several cellular signaling pathways, including TGF $\beta$, cell-communication, focal-adhesion, MAPK, and cell-cycle pathways, which are critically involved in cell growth, survival, migration, and invasion. Furthermore, LSD1 inhibits the invasion of breast cancer cells in a Mi-2-dependent manner in vitro and suppresses breast cancer metastatic potential in vivo. In fact, LSD1 is downregulated in breast carcinomas and negatively correlated with $T G F \beta 1$ expression.

Collectively, the LSD1/NuRD complex is required for the repression of important genes in cellular signaling pathways and suppresses breast cancer metastasis. While LSD1 is a component of NuRD complexes, it has been also observed that LSD1 targets promoter regions independent of NuRD [60]. Therefore, it is likely that LSD1 also functions as a component of currently unidentified protein complexes.

\section{The CoREST Complex}

CoREST was first identified as a corepressor of REST (RE1 Silencing Transcription Factor, also known as NRSF) [61] and later demonstrated to be a component of 


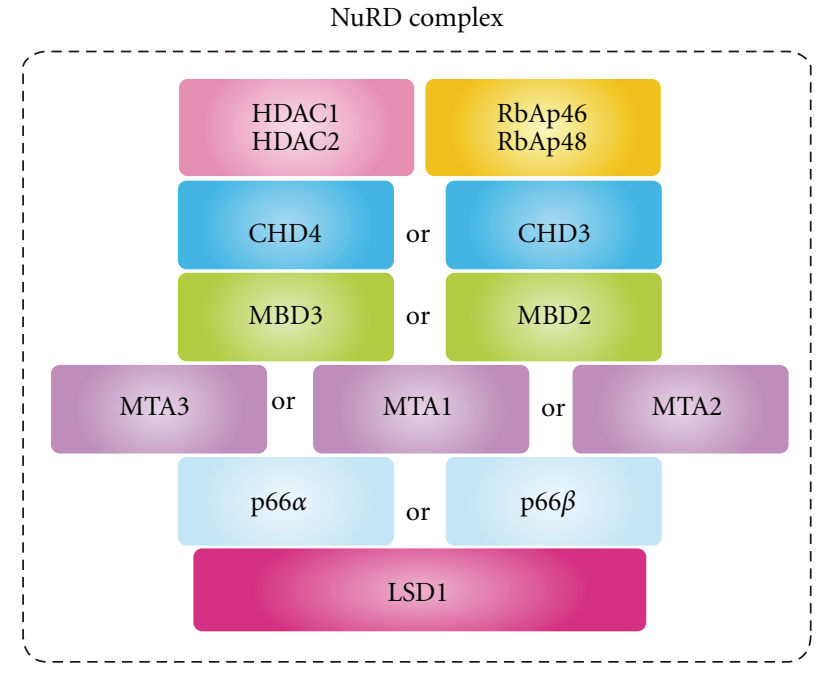

(a)

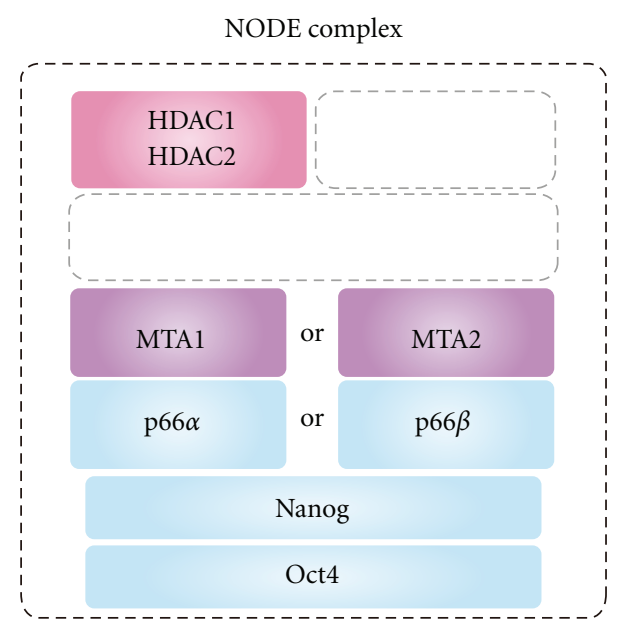

(b)

Figure 2: The NuRD complexes. Schematic representation of the NuRD complex (a) and ES cell-specific NODE complex (b). The empty dotted boxes indicate missing subunits compared with the NuRD complex.

HDAC1/2-containing complexes [62]. The CoREST complex contains HDAC1/2, p80, Sox-like protein, ZNF217 (p110a), and LSD1 (p110b) (Figure 3(a)). Although p110b was identified in the first purification study, its catalytic activity was later identified and renamed LSD1 [6]. Because the CoREST complex does not associate with mSin3, MTA, CHD4, or RbAp46/48, it is thought to be distinct from the other HDAC-containing complexes. The CoREST protein contains two SANT domains, a conserved domain resembling the DNA-binding domains of Myb-related DNAbinding proteins. This domain was originally identified in SWI3, ADA3, NCoR, and TFIIIB (and thus named SANT) [63], and it is also present in the MTA proteins. Interestingly, the demethylase activity of LSD1 to the nucleosomal substrates requires the CoREST protein [64]. In addition, the CoREST/LSD1 complex shows high demethylase activity for a hypoacetylated nucleosome substrate, and the SANT domain of CoREST preferentially interacts with hypoacetylated histone tails, as was also previously observed for the SANT domain of SMRT [65]. These results suggest that the HDACs in the CoREST/LSD1 complex function upstream of LSD1 and that deacetylated states are recognized by CoREST, which facilitates LSD1's enzymatic activity.

ZNF217, the other CoREST complex component, is a Krüppel-like zinc-finger protein. The ZNF217 complex purified from HeLa cells includes LSD1, CoREST, HDAC2, and CtBP [66]. The zinc-finger motif of ZNF217 specifically binds to the DNA sequence CAGAAY ( $\mathrm{Y}$ means $\mathrm{C}$ or T), and this consensus is highly conserved in the E-cadherin promoter. In fact, ZNF217 and other CoREST complex components are found on the E-cadherin promoter, which is repressed by ZNF217. As ZNF217 overexpression has been noted in many cancer cell lines [66], aberrant protein levels of ZNF217 may cause unregulated targeting by the CoRESTLSD1 complex, with a profound effect on cancer progression.
The transcriptional corepressor CtBP (C-terminal binding protein) is also implicated in tumorigenesis. Immunoaffinity purification of the CtBP complex revealed that it contains all of the CoREST complex components [67]. In this analysis, many other interactors were also identified (G9a, HuHMT, HPC2, REBB-1, and ZNF516) [67]. As described for ZNF217, CtBP and the complex components, EuHMT and G9a, also repress E-cadherin promoter activity. Another report showed that Snail, a master regulator of the epithelial-to-mesenchymal transition, forms a complex with LSD1 and CoREST, and functions to recruit LSD1 activity to the E-cadherin promoter [68]. Together, these results suggest that the CoREST complex is a kind of "core complex" that associates with the CtBP complex as an effector module (Figure 3(a)). Considering that CoREST also associates with other corepressors required for hematopoietic differentiation, Gfi-1 and Gfil-b [69], it is likely that CoREST forms several distinct complexes depending on the cellular situation.

\section{The NCoR/SMRT Complex}

Nuclear hormone receptors are evolutionally conserved, ligand-dependent transcription factors that influence the biological processes of cell proliferation and differentiation in metazoans. NCoR (Nuclear receptor CoRepressor, also known as NCOR1) and SMRT (Silencing Mediator for Retinoid and Thyroid receptor, also known as NCOR2) bind to nuclear hormone receptors and act as "platform proteins" in recruiting a large protein complex that includes Class I and II HDAC and $\mathrm{mSin} 3 \mathrm{~A}$ [70]. Of note, proteins homologous to NCoR or SMRT are not found in the yeast genome, as is the case for the nuclear hormone receptors. These proteins appear to have arisen during the evolution of metazoan organisms to allow unliganded nuclear hormone receptors 


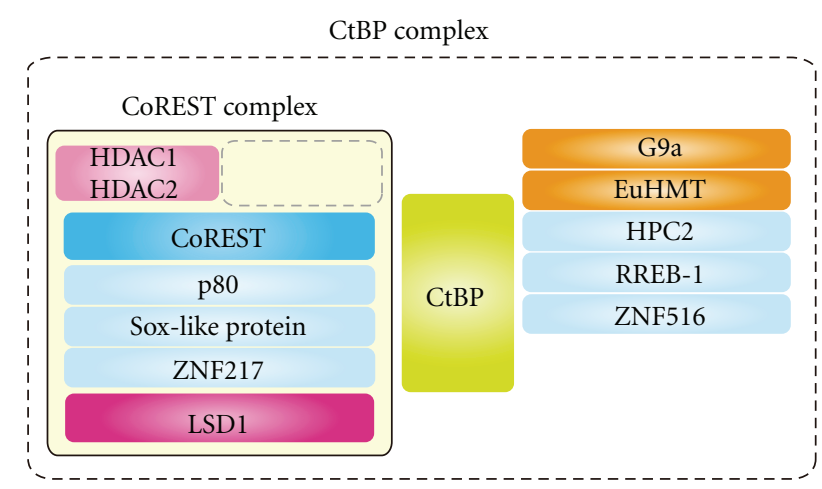

(a)

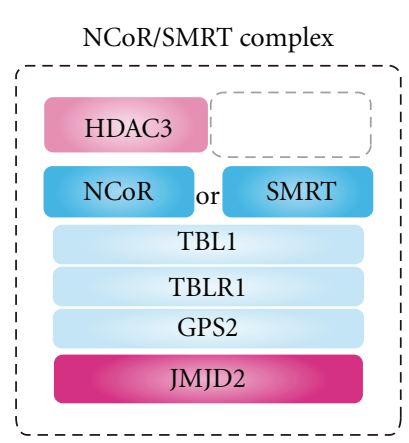

(b)

FIgure 3: The CoREST and NCoR/SMRT complexes. Schematic representation of the CoREST (a) and the NCoR/SMRT (b) complexes. The CoREST complex is included in the CtBP-containing complexes. The empty dotted boxes indicate that both CoREST and NCoR/SMRT complexes lack RbAp46/RbAp48 subunits, which are widely observed in HDAC-containing complexes.

to repress transcription via the Sin3-HDAC pathway [70]. SMRT and NCoR share a similar domain organization and are thought to be paralogues.

NCoR and SMRT are essential for development, but their mutant phenotypes are quite different. NCoR appears to be critical to neural differentiation and the developmental progression of erythrocytes and thymocytes [71, 72], whereas SMRT is required for heart development [72]. The SMRT and NCoR complexes share interacting partners, including HDAC3, TBL1 (transducin $\beta$-like 1), TBLR1 (TBL related 1), and GPS2 (G-protein pathway suppressor 2) (Figure 3(b)) (reviewed in [73]). Moreover, 114 and 98 proteins, respectively, are reported to interact physically with NcoR and SMRT (BioGRID). NCoR and SMRT also associate with other HDAC members, HDAC4, HDAC5, and HDAC7, but these enzymatic activities are dependent on the SMRT/NCoR-HDAC3 complex [74]. Previous studies demonstrated that diverse transcription factors, including Mad/Mxi, BCL6/LAZ3, ETO, RBP-J, and REST/NRSF, interact with the NCoR and SMRT complexes $[75,76]$.

Several demethylases that interact with NCoR have been identified. KDM4A (former name, JMJD2/ JDM3A/JMJD2A/KIAA0677) was identified in an affinitypurified NCoR complex [77]. KDM4A is not a core subunit of the NCoR complex, and it is not required for NCoR-mediated repression by thyroid hormone receptor. However, KDM4A is required for selective repression of the ASCL2 gene, which is an imprinted gene essential for proper placental development, and this repression requires a functional NCoR complex [78]. KDM4A specifically demethylates tri- and dimethylated lysine- 9 and lysine-36 of histone $\mathrm{H} 3$ (H3K9/36 me3/2) [79]. A recent study showed that KDM4 also demethylates lysine-26 of histone H1.4 (H1.4K26me), which is one of seven somatic H1 isotypes in humans, and is known to cause transcriptional repression [80]. Considering that both $\mathrm{H} 3 \mathrm{~K} 9 \mathrm{me} 3 / 2$ and H1.4K26me are repressive histone marks, it is unclear how KDM4's enzymatic activity is coupled with NCoR-mediated repression. Nevertheless, the substrate specificity of KDM4 may be modulated within N-CoR-containing complexes.
Further studies are required to elucidate the function of the KDM4-associated NCoR complex and the resulting histone-modification dynamics.

KDM5C (also known as SMCX/JARID1C) has histone H3K4-specific demethylase activity and functions as a transcriptional repressor. KDM5C is implicated in X-linked mental retardation, epilepsy, and autism [81-83]. Tahiliani et al., purified a KDM5C-containing complex from HeLa cells and identified NCoR [84]. This complex also contains HDAC1/2, the H3K9-specific methyltransferase G9a, REST (RE-1 Silencing Transcription factor, also known as NRSF), which represses neuronal genes in non-neuronal tissues, along with other proteins that are primarily involved in transcriptional repression. REST not only maintains the transcriptional silencing of a range of neuronal genes in differentiated non-neuronal cells but also plays key roles during lineage commitment in neurogenesis (reviewed in [85]). Tahiliani et al., further showed that KDM5C functions at a subset of REST-regulated neuronal specific genes [84]. It should be noted that NCoR was previously shown to form a stable complex with HDAC3, TBL1, TBLR1, and GPS2 [73], but these proteins were not identified in the KDM5C-containing complex. Thus, it is not clear how NCoR functions in the KDM5C-REST complex. However, a requirement for $\mathrm{NCoR}$ in astroglia differentiation was clearly demonstrated [71]. REST modulates not only neuronal, but also glial lineage elaboration [86]. Therefore, it is possible that a unique NCoR-SMCX-REST complex specifically functions in glial development.

Despite its similarity to the NCoR complex, there is no report showing a direct interaction between the SMRT complex and HDMs. However, SMRT appears to control HDM function in trans. SMRT knockout mice exhibit embryonic lethality, with impaired neural development as well as heart defects [72]. SMRT represses the expression of the gene encoding an H3K27-specific demethylase KDM6B (JMJD3), which is also a direct target of the retinoic acid receptor. Retinoic acid induction leads to the release of SMRT from the KDM6B gene promoter, and then KDM6B derepresses neurogenic genes in neural stem cells. This finding uncovered 
a novel hierarchical relationship between the activities of SMRT and HDM in maintaining the neural stem-cell state.

\section{Conclusion}

Many HDACs do not function by themselves but act as a component in a multiprotein complex. This complex formation appears to help each HDAC to exert its catalytic activity more effectively and/or specifically. As described above, most HDAC-containing complexes are associated with histone demethylases (HDMs), and the cooperative action of these enzymatic activities enables the complex to act on multiply modified histones at transcriptionally repressive gene regions. Incorporating subtype components gives rise to variations that allow these complexes to repress a wide variety of target genes. Below, we summarize the points raised in this paper.

(1) The two mammalian Sin3 complexes have distinct functions. In embryonic development, $\mathrm{mSin} 3 \mathrm{~A}$ is required during the preimplantation stage, whereas $\mathrm{mSin} 3 \mathrm{~B}$ is required in late gestation. In particular, $\mathrm{mSin} 3 \mathrm{~B}$ is essential for erythrocyte and granulocyte differentiation.

(2) S. cerevisiae has two Sin3-containing complexes, Rpd3L and Rpd3S. Rpd3L is required for transcriptional repression at gene promoter regions, whereas Rpd3S is located at transcribed regions and prevents spurious transcription from incorrect transcription start sites.

(3) The Drosophila KDM5 protein LID is associated with the SIN3 complex. The LID-SIN3 complex functions to repress the Notch-signaling pathway. Similarly, the human KDM5A protein, RBP2, is required to repress genes of the Notch-signaling pathway.

(4) Two types of MBD protein, MBD2 and MBD3, are distinctively involved in the NuRD complex. $\mathrm{Mbd} 3 / \mathrm{NuRD}$, but not $\mathrm{Mbd} 2 / \mathrm{NuRD}$, is essential for mouse development. Another NuRD component, MTA1/MTA2/MTA3, also contributes to produce distinct complexes. NuRD complexes with each MTA protein bind to specific promoter regions.

(5) Mouse embryonic stem cells contain a specialized NuRD-related complex, NODE. The stem-cellspecific factors Nanog and Oct4 are associated with the NODE complex, which represses several differentiation marker genes.

(6) LSD1 (KDM1A) is associated with the NuRD complex. There are three distinct LSD1-NuRD complexes, LSD1/MTA1/NuRD, LSD1/MTA2/NuRD, and LSD1/MTA3/NuRD. These three different complexes target distinct yet overlapping sets of genes.

(7) LSD1 is also associated with the CoREST complex. Furthermore, all the CoREST complex components are included in the CtBP-containing complex. Many of these components are required for the repression of the E-cadherin promoter.
(8) NCoR/SMRT is the transcriptional repression machinery for nuclear-receptor-mediated transcription. NCoR has critical roles in neural differentiation and hematopoiesis, whereas SMRT is required for heart development. KDM4A is associated with the NCoR complex, and its association is required for ASCL2 gene repression. The NCoR complex also binds SMCX (KDM5C), and the NCoR-SMCX-REST complex functions in glial development.

The transcriptional repression machinery is highly elaborate, and we still lack a complete picture of the transcriptional repression mechanisms. Future studies will continue to uncover these complicated mechanisms.

\section{Acknowledgments}

The authors thank their laboratory members at the RIKEN CDB for helpful discussions and S. Seno for excellent secretarial work. This research was supported by Grant-inAid from the Ministry of Education, Culture, Sports, Science, and Technology of Japan.

\section{References}

[1] B. D. Strahl and C. D. Allis, "The language of covalent histone modifications," Nature, vol. 403, no. 6765, pp. 41-45, 2000.

[2] T. Kouzarides, "Chromatin modifications and their function," Cell, vol. 128, no. 4, pp. 693-705, 2007.

[3] J. B. Rayman, Y. Takahashi, V. B. Indjeian et al., "E2F mediates cell cycle-dependent transcriptional repression in vivo by recruitment of an $\mathrm{HDAC} 1 / \mathrm{mSin} 3 \mathrm{~B}$ corepressor complex," Genes and Development, vol. 16, no. 8, pp. 933-947, 2002.

[4] E. Balciunaite, A. Spektor, N. H. Lents et al., "Pocket protein complexes are recruited to distinct targets in quiescent and proliferating cells," Molecular and Cellular Biology, vol. 25, no. 18, pp. 8166-8178, 2005.

[5] J. Liang, M. Wan, Y. Zhang et al., "Nanog and Oct4 associate with unique transcriptional repression complexes in embryonic stem cells," Nature Cell Biology, vol. 10, no. 6, pp. 731739, 2008.

[6] Y. Shi, F. Lan, C. Matson et al., "Histone demethylation mediated by the nuclear amine oxidase homolog LSD1," Cell, vol. 119, no. 7, pp. 941-953, 2004.

[7] R. J. Klose, Q. Yan, Z. Tothova et al., "The retinoblastoma binding protein RBP2 is an H3K4 demethylase," Cell, vol. 128, no. 5, pp. 889-900, 2007.

[8] P. A. C. Cloos, J. Christensen, K. Agger et al., "The putative oncogene GASC1 demethylates tri- and dimethylated lysine 9 on histone H3," Nature, vol. 442, no. 7100, pp. 307-311, 2006.

[9] K. Agger, P. A. C. Cloos, J. Christensen et al., "UTX and JMJD3 are histone H3K27 demethylases involved in HOX gene regulation and development," Nature, vol. 449, no. 7163, pp. 731-734, 2007.

[10] F. Lan, P. E. Bayliss, J. L. Rinn et al., "A histone H3 lysine 27 demethylase regulates animal posterior development," Nature, vol. 449, no. 7163, pp. 689-694, 2007.

[11] R. J. Klose and Y. Zhang, "Regulation of histone methylation by demethylimination and demethylation," Nature Reviews Molecular Cell Biology, vol. 8, no. 4, pp. 307-318, 2007. 
[12] Y. Zhang, R. Iratni, H. Erdjument-Bromage, P. Tempst, and D. Reinberg, "Histone deacetylases and SAP18, a novel polypeptide, are components of a human Sin3 complex," Cell, vol. 89, no. 3, pp. 357-364, 1997.

[13] Y. Zhang, Z.-W. Sun, R. Iratni et al., "SAP30, a novel protein conserved between human and yeast, is a component of a histone deacetylase complex," Molecular Cell, vol. 1, no. 7, pp. 1021-1031, 1998.

[14] Y. Zhang, G. LeRoy, H.-P. Seelig, W. S. Lane, and D. Reinberg, "The dermatomyositis-specific autoantigen Mi2 is a component of a complex containing histone deacetylase and nucleosome remodeling activities," Cell, vol. 95, no. 2, pp. 279289, 1998.

[15] J.-H. Dannenberg, G. David, S. Zhong, J. Van Der Torre, W. H. Wong, and R. A. DePinho, "mSin3A corepressor regulates diverse transcriptional networks governing normal and neoplastic growth and survival," Genes and Development, vol. 19, no. 13, pp. 1581-1595, 2005.

[16] G. David, K. B. Grandinetti, P. M. Finnerty, N. Simpson, G. C. Chu, and R. A. DePinho, "Specific requirement of the chromatin modifier mSin3B in cell cycle exit and cellular differentiation," Proceedings of the National Academy of Sciences of the United States of America, vol. 105, no. 11, pp. 4168-4172, 2008.

[17] M. J. Carrozza, B. Li, L. Florens et al., "Histone H3 methylation by Set 2 directs deacetylation of coding regions by Rpd3S to suppress spurious intragenic transcription," Cell, vol. 123, no. 4, pp. 581-592, 2005.

[18] M.-C. Keogh, S. K. Kurdistani, S. A. Morris et al., "Cotranscriptional set2 methylation of histone $\mathrm{H} 3$ lysine 36 recruits a repressive Rpd3 complex," Cell, vol. 123, no. 4, pp. 593-605, 2005.

[19] M. L. Youdell, K. O. Kizer, E. Kisseleva-Romanova et al., "Roles for Ctk1 and Spt6 in regulating the different methylation states of histone H3 lysine 36," Molecular and Cellular Biology, vol. 28, no. 16, pp. 4915-4926, 2008.

[20] B. Sun, J. Hong, P. Zhang et al., "Molecular basis of the interaction of Saccharomyces cerevisiae Eaf3 chromo domain with methylated H3K36," Journal of Biological Chemistry, vol. 283, no. 52, pp. 36504-36512, 2008.

[21] C. Xu, G. Cui, M. V. Botuyan, and G. Mer, "Structural basis for the recognition of methylated histone H3K36 by the Eaf3 subunit of histone deacetylase complex Rpd3S," Structure, vol. 16, no. 11, pp. 1740-1750, 2008.

[22] B. Li, M. Gogol, M. Carey, D. Lee, C. Seidel, and J. L. Workman, "Combined action of PHD and chromo domains directs the Rpd3S HDAC to transcribed chromatin," Science, vol. 316, no. 5827, pp. 1050-1054, 2007.

[23] M. J. Bertram, N. G. Bérubé, X. H. Swanson, and O. M. Pereira-Smith, "Assembly of a BAC contig of the complementation group B cell senescence gene candidate region at $4 \mathrm{q} 33$ q34.1 and identification of expressed sequences," Genomics, vol. 56, no. 3, pp. 353-354, 1999.

[24] M. J. Bertram and O. M. Pereira-Smith, "Conservation of the MORF4 related gene family: identification of a new chromo domain subfamily and novel protein motif," Gene, vol. 266, no. 1-2, pp. 111-121, 2001.

[25] G. S. Yochum and D. E. Ayer, "Role for the mortality factors MORF4, MRGX, and MRG15 in transcriptional repression via associations with Pf1, $\mathrm{mSin} 3 \mathrm{~A}$, and transducin-like enhancer of split," Molecular and Cellular Biology, vol. 22, no. 22, pp. 7868-7876, 2002.
[26] T. Hayakawa, Y. Ohtani, N. Hayakawa et al., "RBP2 is an MRG15 complex component and down-regulates intragenic histone H3 lysine 4 methylation," Genes to Cells, vol. 12, no. 6, pp. 811-826, 2007.

[27] Y. Cai, J. Jin, C. Tomomori-Sato et al., "Identification of new subunits of the multiprotein mammalian TRRAP/TIP60containing histone acetyltransferase complex," Journal of Biological Chemistry, vol. 278, no. 44, pp. 42733-42736, 2003.

[28] Y. Doyon, W. Selleck, W. S. Lane, S. Tan, and J. Côté, "Structural and functional conservation of the NuA4 histone acetyltransferase complex from yeast to humans," Molecular and Cellular Biology, vol. 24, no. 5, pp. 1884-1896, 2004.

[29] T. Hayakawa, F. Zhang, N. Hayakawa et al., "MRG15 binds directly to PALB2 and stimulates homology-directed repair of chromosomal breaks," Journal of Cell Science, vol. 123, no. 7, pp. 1124-1130, 2010.

[30] S. M.-H. Sy, M. S. Y. Huen, and J. Chen, "MRG15 is a novel PALB2-interacting factor involved in homologous recombination," Journal of Biological Chemistry, vol. 284, no. 32, pp. 21127-21131, 2009.

[31] J.-I. Nakayama, G. Xiao, K.-I. Noma et al., “Alp13, an MRG family protein, is a component of fission yeast Clr6 histone deacetylase required for genomic integrity," EMBO Journal, vol. 22, no. 11, pp. 2776-2787, 2003.

[32] T. Kusch, L. Florens, W. H. MacDonald et al., "Acetylation by Tip60 is required for selective histone variant exchange at DNA lesions," Science, vol. 306, no. 5704, pp. 2084-2087, 2004.

[33] S. N. Garcia, B. M. Kirtane, A. J. Podlutsky, O. M. PereiraSmith, and K. Tominaga, "Mrg15 null and heterozygous mouse embryonic fibroblasts exhibit DNA-repair defects post exposure to gamma ionizing radiation," FEBS Letters, vol. 581, no. 27, pp. 5275-5281, 2007.

[34] C. van Oevelen, J. Wang, P. Asp et al., "A role for mammalian Sin3 in permanent gene silencing," Molecular Cell, vol. 32, no. 3, pp. 359-370, 2008.

[35] N. Lee, H. Erdjument-Bromage, P. Tempst, R. S. Jones, and Y. Zhang, "The H3K4 demethylase lid associates with and inhibits histone deacetylase Rpd3," Molecular and Cellular Biology, vol. 29, no. 6, pp. 1401-1410, 2009.

[36] Y. M. Moshkin, T. W. Kan, H. Goodfellow et al., "Histone chaperones ASF1 and NAP1 differentially modulate removal of active histone marks by LID-RPD3 complexes during NOTCH silencing," Molecular Cell, vol. 35, no. 6, pp. 782-793, 2009.

[37] S. J. Bray, "Notch signalling: a simple pathway becomes complex," Nature Reviews Molecular Cell Biology, vol. 7, no. 9, pp. 678-689, 2006.

[38] U. Koch and F. Radtke, "Notch and cancer: a double-edged sword," Cellular and Molecular Life Sciences, vol. 64, no. 21, pp. 2746-2762, 2007.

[39] R. Liefke, F. Oswald, C. Alvarado et al., "Histone demethylase KDM5A is an integral part of the core Notch-RBP-J repressor complex," Genes and Development, vol. 24, no. 6, pp. 590-601, 2010.

[40] L. A. Pile, P. T. Spellman, R. J. Katzenberger, and D. A. Wassarman, "The SIN3 deacetylase complex represses genes encoding mitochondrial proteins: implications for the regulation of energy metabolism," Journal of Biological Chemistry, vol. 278, no. 39, pp. 37840-37848, 2003.

[41] N. Lopez-Bigas, T. A. Kisiel, D. C. DeWaal et al., "Genomewide analysis of the $\mathrm{H} 3 \mathrm{~K} 4$ histone demethylase RBP2 reveals a transcriptional program controlling differentiation," Molecular Cell, vol. 31, no. 4, pp. 520-530, 2008. 
[42] J. K. Tong, C. A. Hassig, G. R. Schnitzler, R. E. Kingston, and S. L. Schreiber, "Chromatin deacetylation by an ATP-dependent nucleosome remodelling complex," Nature, vol. 395, no. 6705, pp. 917-921, 1998.

[43] Y. Xue, J. Wong, G. T. Moreno, M. K. Young, J. Côté, and W. Wang, "NURD, a novel complex with both ATP-dependent chromatin-remodeling and histone deacetylase activities," Molecular Cell, vol. 2, no. 6, pp. 851-861, 1998.

[44] Q. Ge, D. S. Nilasena, C. A. O'Brien, M. B. Frank, and I. N. Targoff, "Molecular analysis of a major antigenic region of the $240-\mathrm{kD}$ protein of Mi-2 autoantigen," Journal of Clinical Investigation, vol. 96, no. 4, pp. 1730-1737, 1995.

[45] M. Saito and F. Ishikawa, "The mCpG-binding domain of human MBD3 does not bind to $\mathrm{mCpG}$ but interacts with NuRD/Mi2 components HDAC1 and MTA2," Journal of Biological Chemistry, vol. 277, no. 38, pp. 35434-35439, 2002.

[46] B. Hendrich and A. Bird, "Identification and characterization of a family of mammalian methyl-CpG binding proteins," Molecular and Cellular Biology, vol. 18, no. 11, pp. 6538-6547, 1998.

[47] Y. Zhang, H.-H. Ng, H. Erdjument-Bromage, P. Tempst, A. Bird, and D. Reinberg, "Analysis of the NuRD subunits reveals a histone deacetylase core complex and a connection with DNA methylation," Genes and Development, vol. 13, no. 15, pp. 1924-1935, 1999.

[48] X. Le Guezennec, M. Vermeulen, A. B. Brinkman et al., "MBD2/NuRD and MBD3/NuRD, two distinct complexes with different biochemical and functional properties," Molecular and Cellular Biology, vol. 26, no. 3, pp. 843-851, 2006.

[49] B. Hendrich, J. Guy, B. Ramsahoye, V. A. Wilson, and A. Bird, "Closely related proteins MBD2 and MBD3 play distinctive but interacting roles in mouse development," Genes and Development, vol. 15, no. 6, pp. 710-723, 2001.

[50] R. Kumar, R.-A. Wang, A. Mazumdar et al., "A naturally occurring MTA1 variant sequesters oestrogen receptor- $\alpha$ in the cytoplasm," Nature, vol. 418, no. 6898, pp. 654-657, 2002.

[51] N. Fujita, D. L. Jaye, M. Kajita, C. Geigerman, C. S. Moreno, and P. A. Wade, "MTA3, a Mi-2/NuRD complex subunit, regulates an invasive growth pathway in breast cancer," Cell, vol. 113, no. 2, pp. 207-219, 2003.

[52] Y. Toh, E. Oki, S. Oda et al., "Overexpression of the MTA1 gene in gastrointestinal carcinomas: correlation with invasion and metastasis," International Journal of Cancer, vol. 74, no. 4, pp. 459-463, 1997.

[53] Y. Toh, H. Kuwano, M. Mori, G. L. Nicolson, and K. Sugimachi, "Overexpression of metastasis-associated MTA1 mRNA in invasive oesophageal carcinomas," British Journal of Cancer, vol. 79, no. 11-12, pp. 1723-1726, 1999.

[54] A. Mazumdar, R.-A. Wang, S. K. Mishra et al., "Transcriptional repression of oestrogen receptor by metastasisassociated protein 1 corepressor," Nature Cell Biology, vol. 3, no. 1, pp. 30-37, 2001.

[55] K. Kaji, I. M. Caballero, R. MacLeod, J. Nichols, V. A. Wilson, and $\mathrm{B}$. Hendrich, "The NuRD component Mbd3 is required for pluripotency of embryonic stem cells," Nature Cell Biology, vol. 8, no. 3, pp. 285-292, 2006.

[56] K. Kaji, J. Nichols, B. Hendrich, and B. Hendrich, "Mbd3, a component of the NuRD co-repressor complex, is reqiured for development of pluripotent cells," Development, vol. 134, no. 6, pp. 1123-1132, 2007.

[57] M. Brackertz, Z. Gong, J. Leers, and R. Renkawitz, "p66 $\alpha$ and p $66 \beta$ of the Mi-2/NuRD complex mediate MBD2 and histone interaction," Nucleic Acids Research, vol. 34, no. 2, pp. 397-406, 2006.
[58] S. Marino and R. Nusse, "Mutants in the mouse NuRD/Mi2 component P66alpha are embryonic lethal," PloS One, vol. 2, no. 6, article e519, 2007.

[59] I. Chambers and A. Smith, "Self-renewal of teratocarcinoma and embryonic stem cells," Oncogene, vol. 23, no. 43, pp. 71507160, 2004.

[60] Y. Wang, H. Zhang, Y. Chen et al., "LSD1 is a subunit of the NuRD complex and targets the metastasis programs in breast cancer," Cell, vol. 138, no. 4, pp. 660-672, 2009.

[61] M. E. Andres, C. Burger, M. J. Peral-Rubio et al., "CoREST: a functional corepressor required for regulation of neuralspecific gene expression," Proceedings of the National Academy of Sciences of the United States of America, vol. 96, no. 17, pp. 9873-9878, 1999.

[62] A. You, J. K. Tong, C. M. Grozinger, and S. L. Schreiber, "CoREST is an integral component of the CoREST-human histone deacetylase complex," Proceedings of the National Academy of Sciences of the United States of America, vol. 98, no. 4, pp. 1454-1458, 2001.

[63] R. Aasland, A. F. Stewart, and T. Gibson, "The SANT domain: a putative DNA-binding domain in the SWI-SNF and ADA complexes, the transcriptional co-repressor N-CoR and TFIIIB," Trends in Biochemical Sciences, vol. 21, no. 3, pp. 87$88,1996$.

[64] Y.-J. Shi, C. Matson, F. Lan, S. Iwase, T. Baba, and Y. Shi, "Regulation of LSD1 histone demethylase activity by its associated factors," Molecular Cell, vol. 19, no. 6, pp. 857-864, 2005.

[65] J. Yu, Y. Li, T. Ishizuka, M. G. Guenther, and M. A. Lazar, "A SANT motif in the SMRT corepressor interprets the histone code and promotes histone deacetylation," EMBO Journal, vol. 22, no. 13, pp. 3403-3410, 2003.

[66] J. J. M. Cowger, Q. Zhao, M. Isovic, and J. Torchia, "Biochemical characterization of the zinc-finger protein 217 transcriptional repressor complex: identification of a ZNF217 consensus recognition sequence," Oncogene, vol. 26, no. 23, pp. 3378-3386, 2007.

[67] Y. Shi, J.-I. Sawada, G. Sui et al., "Coordinated histone modifications mediated by a CtBP co-repressor complex," Nature, vol. 422, no. 6933, pp. 735-738, 2003.

[68] Y. Lin, Y. Wu, J. Li et al., "The SNAG domain of snail1 functions as a molecular hook for recruiting lysine-specific demethylase 1," EMBO Journal, vol. 29, no. 11, pp. 1803-1816, 2010.

[69] S. Saleque, J. Kim, H. M. Rooke, and S. H. Orkin, "Epigenetic regulation of hematopoietic differentiation by Gfi-1 and Gfi$1 \mathrm{~b}$ is mediated by the cofactors CoREST and LSD1," Molecular Cell, vol. 27, no. 4, pp. 562-572, 2007.

[70] P. Ordentlich, M. Downes, W. Xie, A. Genin, N. B. Spinner, and R. M. Evans, "Unique forms of human and mouse nuclear receptor corepressor SMRT," Proceedings of the National Academy of Sciences of the United States of America, vol. 96, no. 6, pp. 2639-2644, 1999.

[71] O. Hermanson, K. Jepsen, and M. G. Rosenfeld, "N-CoR controls differentiation of neural stem cells into astrocytes," Nature, vol. 419, no. 6910, pp. 934-939, 2002.

[72] K. Jepsen, D. Solum, T. Zhou et al., "SMRT-mediated repression of an H3K27 demethylase in progression from neural stem cell to neuron," Nature, vol. 450, no. 7168, pp. 415-419, 2007.

[73] V. Perissi, K. Jepsen, C. K. Glass, and M. G. Rosenfeld, "Deconstructing repression: evolving models of co-repressor action," Nature Reviews Genetics, vol. 11, no. 2, pp. 109-123, 2010. 
[74] W. Fischle, F. Dequiedt, M. J. Hendzel et al., "Enzymatic activity associated with class II HDACs is dependent on a multiprotein complex containing HDAC3 and SMRT/NCoR," Molecular Cell, vol. 9, no. 1, pp. 45-57, 2002.

[75] C. K. Glass and M. G. Rosenfeld, "The coregulator exchange in transcriptional functions of nuclear receptors," Genes and Development, vol. 14, no. 2, pp. 121-141, 2000.

[76] K. Jepsen, O. Hermanson, T. M. Onami et al., "Combinatorial roles of the nuclear receptor corepressor in transcription and development," Cell, vol. 102, no. 6, pp. 753-763, 2000.

[77] H.-G. Yoon, D. W. Chan, A. B. Reynolds, J. Qin, and J. Wong, "N-CoR mediates DNA methylation-dependent repression through a methyl CpG binding protein Kaiso," Molecular Cell, vol. 12, no. 3, pp. 723-734, 2003.

[78] D. Zhang, H.-G. Yoon, and J. Wong, "JMJD2A is a novel NCoR-interacting protein and is involved in repression of the human transcription factor achaete scute-like homologue 2 (ASCL2/Hash2)," Molecular and Cellular Biology, vol. 25, no. 15, pp. 6404-6414, 2005.

[79] S. S. Ng, K. L. Kavanagh, M. A. McDonough et al., "Crystal structures of histone demethylase JMJD2A reveal basis for substrate specificity," Nature, vol. 448, no. 7149, pp. 87-91, 2007.

[80] P. Trojer, J. Zhang, M. Yonezawa et al., "Dynamic histone H1 isotype 4 methylation and demethylation by histone lysine methyltransferase G9a/KMT1C and the jumonji domaincontaining JMJD2/KDM4 proteins," Journal of Biological Chemistry, vol. 284, no. 13, pp. 8395-8405, 2009.

[81] A. Tzschach, S. Lenzner, B. Moser et al., "Novel JARID1C/SMCX mutations in patients with X-linked mental retardation," Human Mutation, vol. 27, no. 4, p. 389, 2006.

[82] A. Adegbola, H. Gao, S. Sommer, and M. Browning, "A novel mutation in JARID1C/SMCX in a patient with autism spectrum disorder (ASD)," American Journal of Medical Genetics A, vol. 146, no. 4, pp. 505-511, 2008.

[83] L. R. Jensen, M. Amende, U. Gurok et al., "Mutations in the JARID1C gene, which is involved in transcriptional regulation and chromatin remodeling, cause X-linked mental retardation," American Journal of Human Genetics, vol. 76, no. 2, pp. 227-236, 2005.

[84] M. Tahiliani, P. Mei, R. Fang et al., "The histone H3K4 demethylase SMCX links REST target genes to X-linked mental retardation," Nature, vol. 447, no. 7144, pp. 601-605, 2007.

[85] J. M. Coulson, "Transcriptional regulation: cancer, neurons and the REST," Current Biology, vol. 15, no. 17, pp. R665R668, 2005.

[86] J. J. Abrajano, I. A. Qureshi, S. Gokhan, D. Zheng, A. Bergman, and M. F. Mehler, "Differential deployment of REST and CoREST promotes glial subtype specification and oligodendrocyte lineage maturation," PLoS One, vol. 4, no. 11, Article ID e7665, 2009. 

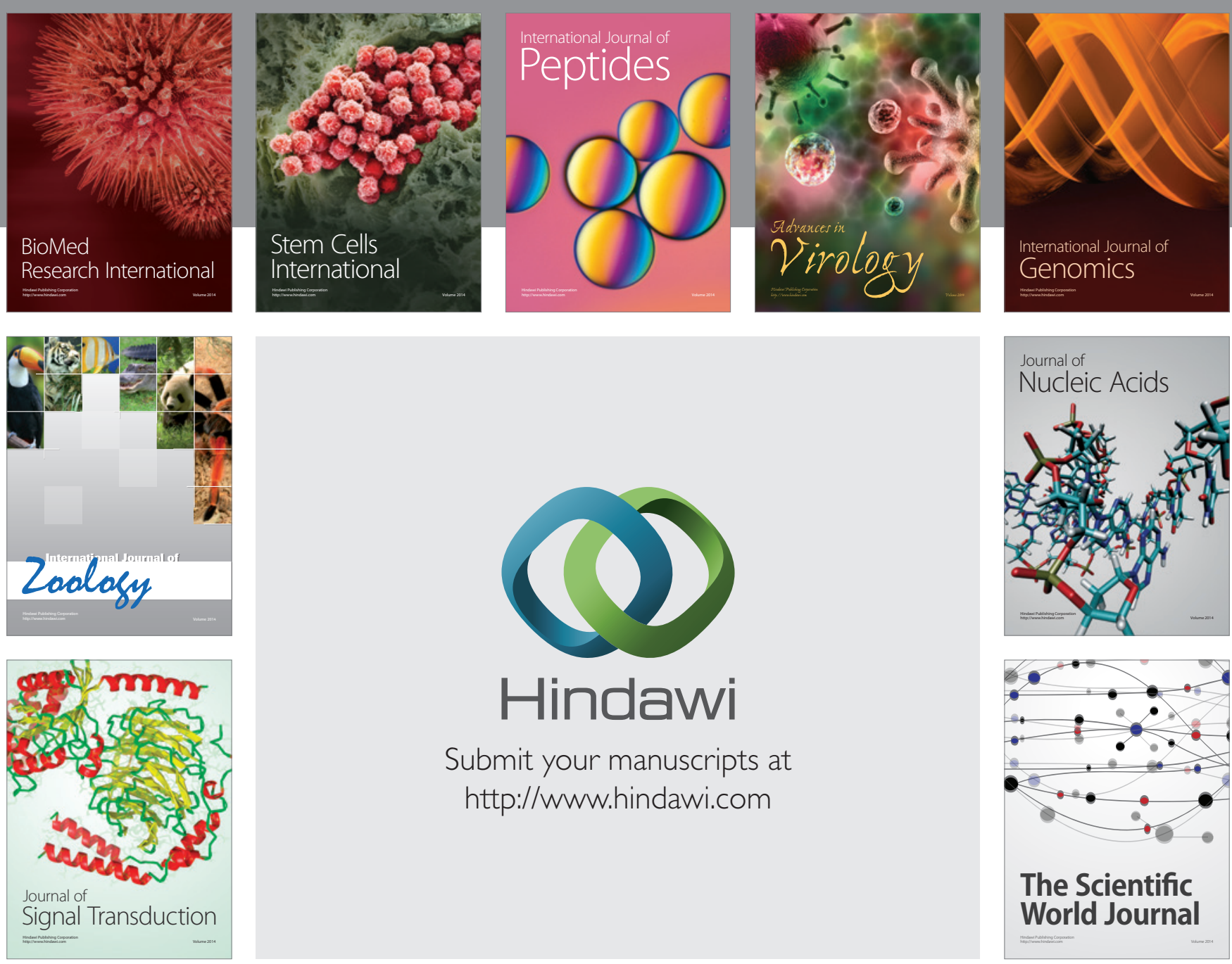

Submit your manuscripts at

http://www.hindawi.com
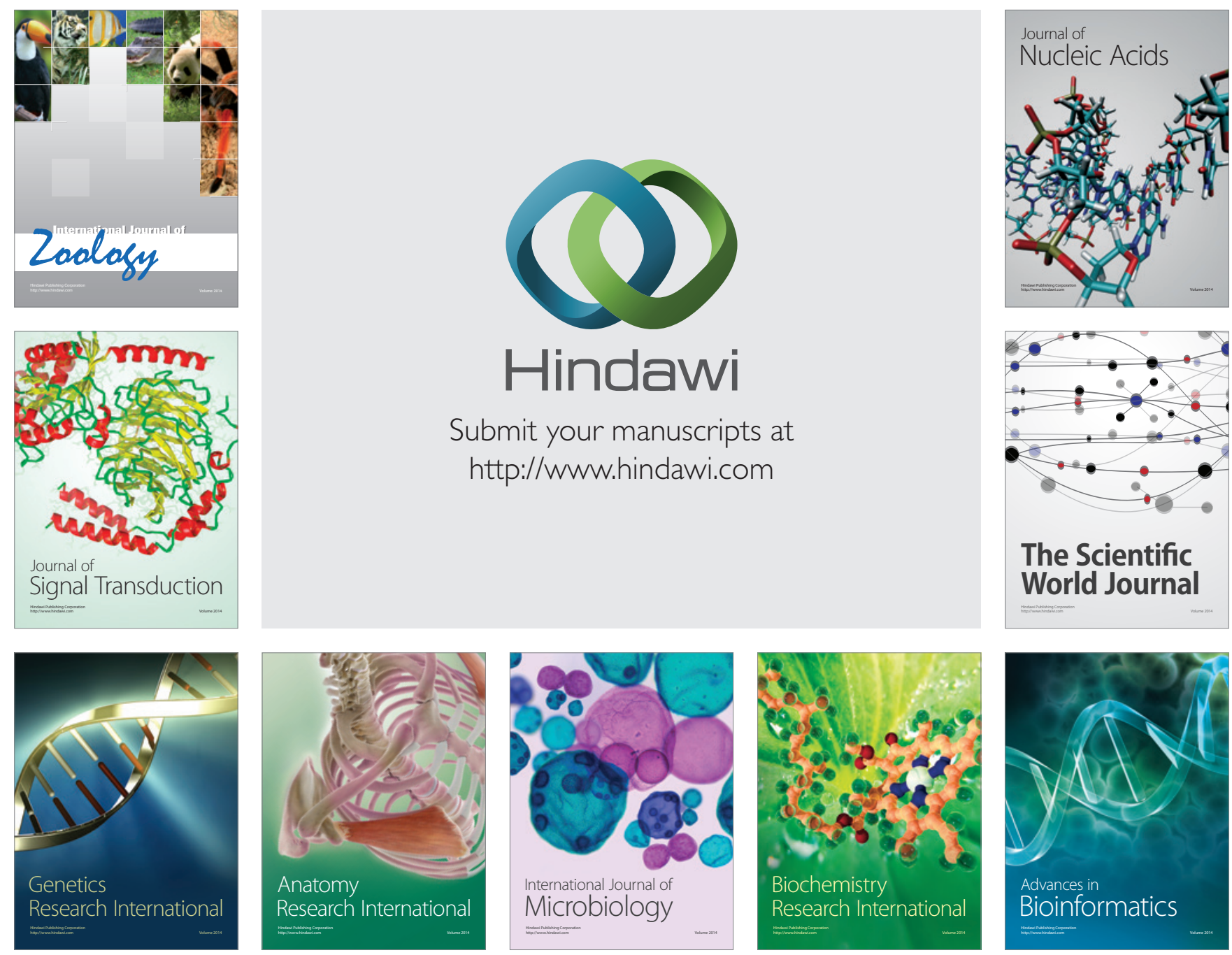

The Scientific World Journal
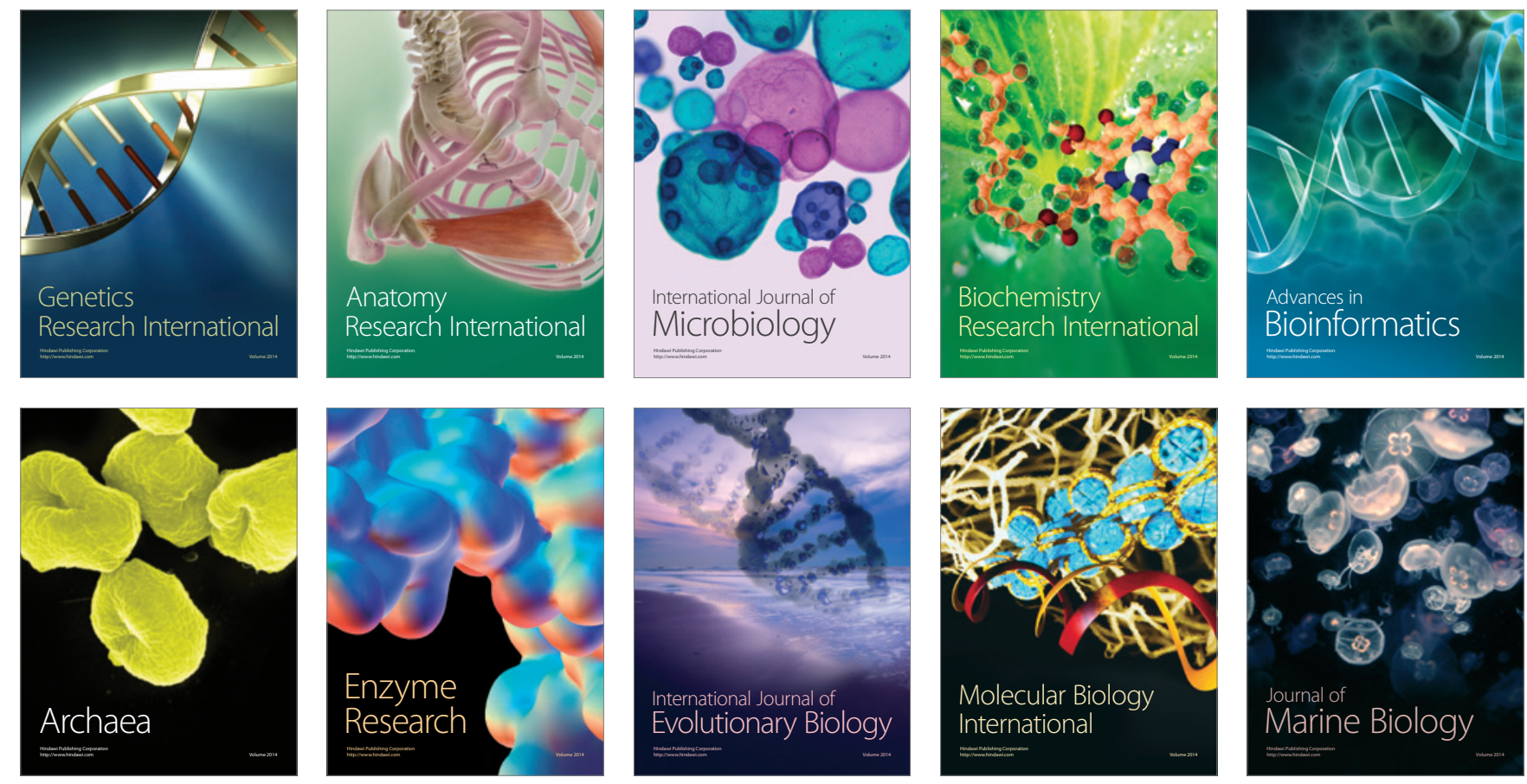